\title{
Efficient in vitro RNA interference and immunofluorescence-based phenotype analysis in a human parasitic nematode, Brugia malayi
}

\author{
Frédéric Landmann ${ }^{1 * \dagger}$, Jeremy M Foster ${ }^{2 \dagger}$, Barton E Slatko ${ }^{2}$ and William Sullivan ${ }^{1}$
}

\begin{abstract}
Background: RNA interference (RNAi) is an efficient reverse genetics technique for investigating gene function in eukaryotes. The method has been widely used in model organisms, such as the free-living nematode Caenorhabditis elegans, where it has been deployed in genome-wide high throughput screens to identify genes involved in many cellular and developmental processes. However, RNAi techniques have not translated efficiently to animal parasitic nematodes that afflict humans, livestock and companion animals across the globe, creating a dependency on data tentatively inferred from C. elegans.

Results: We report improved and effective in vitro RNAi procedures we have developed using heterogeneous short interfering RNA (hsiRNA) mixtures that when coupled with optimized immunostaining techniques yield detailed analysis of cytological defects in the human parasitic nematode, Brugia malayi. The cellular disorganization observed in B. malayi embryos following RNAi targeting the genes encoding $\gamma$-tubulin, and the polarity determinant protein, PAR-1, faithfully phenocopy the known defects associated with gene silencing of their $C$. elegans orthologs. Targeting the B. malayi cell junction protein, AJM-1 gave a similar but more severe phenotype than that observed in C. elegans. Cellular phenotypes induced by our in vitro RNAi procedure can be observed by immunofluorescence in as little as one week.

Conclusions: We observed cytological defects following RNAi targeting all seven B. malayi transcripts tested and the phenotypes mirror those documented for orthologous genes in the model organism C. elegans. This highlights the reliability, effectiveness and specificity of our RNAi and immunostaining procedures. We anticipate that these techniques will be widely applicable to other important animal parasitic nematodes, which have hitherto been mostly refractory to such genetic analysis.
\end{abstract}

Keywords: RNAi, nematode, immunostaining, Brugia, filaria

\section{Background}

Filarial nematodes cause debilitating pathologies in tropical areas with $>1$ billion people at risk. Current anthelmintic drugs predominantly target larval stages only, and a developing resistance has been indicated [1-3]. Since almost all filarial species that cause disease in humans rely on the bacterial endosymbiont Wolbachia for proper embryogenesis, development and viability, these symbionts have become a major drug target

\footnotetext{
* Correspondence: landmann@biology.ucsc.edu

† Contributed equally

'Department of Molecular, Cell and Developmental Biology, University of

California Santa Cruz, 1156 High Street, Santa Cruz, CA 95604, USA

Full list of author information is available at the end of the article
}

for novel anti-filarial strategies $[4,5]$. It is crucial to characterize the molecular and cellular mechanisms underlying Wolbachia transmission, since their segregation patterns in the embryo determine the localization in adult hypodermal chords [6]. Such knowledge could lead to the molecular identification of new drug targets.

Despite the published genomes of Brugia malayi [7], a causative agent of lymphatic filariasis and elephantiasis, and its Wolbachia endosymbiont [8], both the developmental biology of filarial nematodes and the mutualism with Wolbachia are still poorly understood. The free living nematode Caenorhabditis elegans, remains the closest animal model to date. While C. elegans represents a

\section{Ciomed Central}


valid model for general aspects of nematode biology [9], its estimated $>500$ million year separation from $B$. malayi [10] and free-living lifestyle leave questions related to parasitism unanswered [11]. Furthermore, $C$. elegans data cannot inform on mutualism with Wolbachia since within the Nematoda this bacterium appears limited to parasitic nematodes within the family Onchocercidae [4]. Therefore inference of gene function or biological processes in parasitic species based on data from C. elegans should be made with caution. While reverse genetic tools such as RNA interference (RNAi) are routinely used in $C$. elegans research to characterize gene function [12], RNAi experiments in animal parasitic nematodes have proven notoriously challenging [13-18] with few successes reported. A bioinformatic study comparing the RNAi effector protein complements of various animal parasitic nematodes to that of C. elegans found that while quantitative differences exist (with C. elegans having the richest complement), all species were qualitatively similar and should be RNAi-competent [19]. Indeed, there are a few reports of successful RNAi in filarial nematodes [20-26] although several of these studies targeted the same genes. Various explanations for the limited success of RNAi in animal parasitic nematodes have been proposed [15,19,27].

We describe an efficient in vitro RNAi procedure we have developed to successfully copy RNAi-induced phenotypes observed in C. elegans during early embryogenesis in the parasitic nematode B. malayi. We also further optimized our immunofluorescence protocols [6] to permit detailed characterization of the embryonic RNAi phenotypes. These methodologies may be extended to genes expressed in the germ line or in adult tissues. Our results demonstrate that RNAi can be a reliable and effective tool for gene function studies in parasitic species. The enhanced procedures for both RNA delivery and subsequent immunofluorescencebased phenotype analysis open the way to address fundamental questions in parasitic nematode biology, emancipation from the C. elegans model, and investigation of the interaction between the filarial parasite and its Wolbachia symbiont.

\section{Methods}

See Additional file 1 for a detailed step-by-step protocol and Additional files 2, 3, 4 for illustration of key steps in the procedure.

\section{Preparation of heterogeneous short interfering RNA (hsiRNA)}

Total RNA was prepared from adult B. malayi (TRS Labs, Athens, GA, USA) by established methodology http://www.filariasiscenter.org/molecular-resources/protocols and $\sim 700 \mathrm{ng}$ RNA used as template for production of cDNA using the ProtoScript M-MuLV First Strand cDNA Synthesis Kit according to the manufacturer's instructions (New England Biolabs. Ipswich, MA, USA).

DNA templates for in vitro transcription were generated by PCR using Crimson Taq DNA Polymerase (New England Biolabs). PCR primers contained T7 promoter sequence followed by two guanine bases at their 5 ' ends for transcription by T7 RNA polymerase and enhanced transcription yield. Primers were designed to yield a PCR product corresponding to $\sim 500 \mathrm{bp}$ of the transcript selected for gene silencing. Typically a region towards the 5' end of the open reading frame was selected and primers that would span an intron in genomic DNA (gDNA) were designed. This allows differentiation between amplification from $1^{\text {st }}$ strand cDNA and any residual gDNA present in the cDNA preparation. However, we also demonstrated that RNA transcribed from gDNA that included introns was effective in gene silencing (see par-1 example). Primers having potential to amplify other $B$. malayi genes or to generate amplicons with considerable sequence similarity to non-target transcripts were avoided. PCR mixes $(50 \mu \mathrm{l})$ generally contained $\sim 100 \mathrm{ng}$ template cDNA, $0.4 \mu \mathrm{M}$ each primer, $0.2 \mathrm{mM}$ dNTPs and $0.25 \mu \mathrm{l}(1.25 \mathrm{U})$ polymerase in $1 \times$ buffer. Between 1 and $5 \mu \mathrm{l}$ of the PCR product was loaded on to a $1.5 \%$ agarose gel to assess the quality and size of the amplicon.

dsRNA corresponding to the gene to be targeted by RNAi was prepared using the HiScribe T7 In Vitro Transcription Kit according to the recommended protocol (New England Biolabs). Approximately $1 \mu \mathrm{g}$ of PCR product was used in a $80 \mu \mathrm{l}$ reaction without any purification prior to transcription. The reaction was incubated at $42^{\circ} \mathrm{C}$ for 2.5 hours then checked by loading $1 \mu \mathrm{L}$ on a $1.5 \%$ agarose gel alongside dsRNA Ladder-A (New England Biolabs). When suitable RNA products were visualized, the remainder of the sample was purified by isopropanol precipitation and the RNA pellet resuspended in $50 \mu \mathrm{L}$ RNase-free water.

The long dsRNA was processed to heterogeneous short interfering RNA (hsiRNA) using ShortCut RNase III as recommended (New England Biolabs). The entire preparation of dsRNA $(50 \mu \mathrm{l})$ was used in a $100 \mu \mathrm{l}$ reaction. The hsiRNA was purified by ethanol precipitation, resuspended in $60 \mu \mathrm{L}$ RNase-free water, and an aliquot examined by gel electrophoresis (2\% agarose) alongside siRNA Marker (New England Biolabs). Quantification of hsiRNA was achieved using a Nanodrop or standard spectrophotometer.

\section{In vitro hsiRNAi by soaking}

In a laminar flow hood, the appropriate amount of hsiRNA was placed in wells of a 12 -well cell culture 
plate to which $1 \mathrm{ml}$ Worm Culture Medium (WCM) was added. [WCM: RPMI-1640 cell culture medium with L-glutamine (Invitrogen, Carlsbad, CA, USA), 1\% glucose, $100 \times$ antibiotic-antimycotic solution (A-5955; Sigma-Aldrich, St Louis, MO, USA), 10\% fetal bovine serum inactivated at $56^{\circ} \mathrm{C}$ for 30 minutes (Invitrogen)]. Two female worms were transferred to each well using a curved pick (see Additional file 2). The medium was replaced every 12 hours by pipetting hsiRNA into new wells, adding fresh WCM and then transferring the worms (see Additional file 3). Typically $1 \mu \mathrm{M}$ hsiRNA was used but the concentration was increased up to 5 $\mu \mathrm{M}$ in instances where no effect was observed. Controls included worms cultured similarly but in the absence of any RNA and worms cultured in an equivalent concentration of Lit28i polylinker ShortCut siRNA mix (New England Biolabs).

Worms were incubated in a $37^{\circ} \mathrm{C}, 5 \% \mathrm{CO}_{2}$ incubator. Exposure to the hsiRNA mixture was continued for between 2 and 5 days.

\section{Embryo and Tissue Collection and Fixation}

We optimized and simplified our previously published protocol [6]. The worms from each well were transferred to a drop of $10 \mu \mathrm{l} 1 \times$ PBS on a microscope slide using the curved pick. For maximizing recovery of embryos, the worms were diced into small fragments using a razor blade (see Additional file 4). To obtain larger anatomical structures, such as the ovaries, fewer cuts were made under a dissection microscope, close to the posterior tip for instance, since the hydrostatic pressure expels organs out of the pseudocoelom and into the PBS. Once the ovaries, uteri, or intestine were expelled, they were cut into shorter fragments to facilitate subsequent tissue penetration by antibodies. The fragments and embryos were transferred to a microcentrifuge tube by pipetting $180 \mu \mathrm{L} 1 \times$ PBS, $1 \%$ NP-40 across the slide surface and into the tube (see Additional file 4). Next, $20 \mu \mathrm{l} 32 \%$ paraformaldehyde and 2 volumes heptane $(\sim 400 \mu \mathrm{l})$ were added. The tube was vortexed for one minute, then left on a rotator for 20 minutes. The contents were pelleted in a microcentrifuge $(2000 \times g, 1$ $\mathrm{min})$, the supernatant removed and the pellet resuspended in $1 \mathrm{ml}$ PBST-BSA $(1 \times$ PBS, $0.02 \%$ Triton X100, 2\% BSA (Fraction V; Fisher Scientific, Pittsburgh PA, USA)) by rotation for 5 minutes.

For embryo staining specifically, the tube was centrifuged again and the pellet transferred to a microscope slide using a Pasteur pipette. A coverslip was applied and pressed down gently using a paper towel to absorb excess PBST. Using forceps, the slide was held submerged in liquid nitrogen until the liquid stopped boiling. The slide was placed on the bench and the coverslip removed with a razor blade. Once the embryos had thawed they were washed into a microcentrifuge tube by pipetting PBST-BSA over the slide surface (see Additional file 4). This "freeze-crack" step removes a high proportion of the eggshells, thereby increasing the yield of high quality immunostaining.

These protocols were optimized for young embryos and tissue fragments, and permit immunostaining of all embryos up to about the 20 cell stage. However, the proportion of stained embryos decreases with increasing development stage, possibly because of changes in the eggshell composition, that increase resistance to the treatment.

\section{Alternative treatment for fixation of older embryos}

To increase the staining of older embryos (i.e. after morphogenesis begins), the following step was used instead of the "freeze-crack" procedure. After dicing the worms, the tissues/embryos were transferred from the slide to a microcentrifuge tube using $180 \mu \mathrm{l} 1 \times \mathrm{PBS}$, $20 \%$ bleach (see Additional file 4) then vortexed for no more than 30 seconds. PBS $(1 \mathrm{ml})$ was added, the tube shaken then centrifuged immediately $(2000 \times g, 1 \mathrm{~min})$. The supernatant was removed with a pipette then 180 $\mu \mathrm{l} 1 \times \mathrm{PBS}, 1 \% \mathrm{NP}-40$ was added to the pellet followed by $20 \mu \mathrm{l} 32 \%$ paraformaldehyde and 2 volumes heptane $(\sim 400 \mu \mathrm{l})$. The sample was then processed as described above resulting in embryos in PBST-BSA.

\section{RNase treatment (optional)}

When DNA was to be stained with propidium iodide the samples were treated with RNase prior to antibody incubations. The tubes containing samples in PBST were centrifuged $(2000 \times g, 1 \mathrm{~min})$, the supernatant removed, and replaced by $1 \mathrm{ml} 10 \mathrm{mg} / \mathrm{ml}$ RNaseA (Sigma Aldrich, Catalog No. R4875) in PBST. The sample was rotated overnight at $4^{\circ} \mathrm{C}$.

[For subsequent analysis using a fluorescence microscope or a confocal equipped with a UV laser, DNA can alternatively be stained at the end of the procedures with DAPI-containing mounting medium.]

\section{Staining and Immunostaining}

Samples were spun $(2000 \times g, 1 \mathrm{~min})$ to remove PBST (or PBST-RNase), and resuspended in $500 \mu \mathrm{l}$ fresh PBST. Primary antibodies were diluted into the sample, which was then rotated overnight at $4^{\circ} \mathrm{C}$. Next day, the samples were centrifuged $(2000 \times g, 1 \mathrm{~min})$, the supernatant removed and replaced by $1.5 \mathrm{ml}$ PBST and the tube rotated at room temperature for $>15$ minutes. This step was repeated twice but the sample resuspended in $500 \mu \mathrm{l}$ PBST after the final wash. Secondary fluorochrome-conjugated antibodies were added according to the manufacturer's recommended dilution and the samples rotated overnight at $4^{\circ} \mathrm{C}$. The tubes were 
centrifuged $(2000 \times g, 1 \mathrm{~min})$, the supernatant discarded and $1.5 \mathrm{ml}$ PBST added. The tubes were rotated for > 15 minutes then centrifuged again. The supernatant was discarded, $1.5 \mathrm{ml} \mathrm{PBST}$ added and, if required, $20 \mu \mathrm{l}$ of $1 \mathrm{mg} / \mathrm{ml}$ propidium iodide (Invitrogen) added. The tube contents were mixed by shaking for 20 seconds, then centrifuged again. The supernatant was removed and replaced by $1.5 \mathrm{ml}$ PBST. The tube was again shaken for 20 seconds, centrifuged, and as much liquid as possible carefully removed using a pipette. About $30 \mu \mathrm{l}$ mounting medium (e.g. Vectashield, Vector Laboratories, Burlingame, CA, USA) was added to the tube. The mounting medium can contain DAPI if the DNA was not already stained with propidium iodide. The mounting medium and sample were mixed by gently pipetting up and down with a Pasteur pipette then transferred to a microscope slide. At this point, larger adult worm fragments can be discarded or transferred to a second slide using fine tweezers. This reduces the volume between the slide and coverslip, thereby minimizing floatation of embryos during observation. Adult fragments were mounted on a second slide using $30 \mu \mathrm{l}$ (or more) of mounting medium. Coverslips were added and for embryo preparations, downwards pressure was applied using a paper towel to absorb excess mounting medium and stabilize the embryos. Slides were sealed with transparent nail polish and stored at $4^{\circ} \mathrm{C}$ in the dark. When DAPI was used in the mounting medium, the slides were stored for $>24 \mathrm{hr}$ prior to microscopy to allow the stain to penetrate embryos and tissues completely.

\section{Microscopy}

Confocal microscope images were captured on an inverted photoscope (DMIRB; Leica Microsystems, Wetzlar, Germany) equipped with a laser confocal imaging system (TCS SP2; Leica) using an HCX PL APO 1.4 NA 63 oil objective (Leica) at room temperature.

Microtubule stainings were performed using the monoclonal DM1 $\alpha$ antibody raised against $\alpha$-tubulin (Cell Signaling Technology, Danvers, MA, USA) at a dilution of 1:100. A Cy5 goat anti-mouse secondary antibody (Invitrogen) was used at 1:250. Actin stainings were performed using the fluorescent Atto 488 phalloidin (Sigma) at a dilution of 1:100.

\section{Results and discussion}

To test RNAi in B. malayi, we decided to target orthologs of a number of genes known to be involved in $C$. elegans development and which show clear cellular phenotypes when depleted in that species. We specifically targeted proteins with a structural role, and their regulators.

We started with $\gamma$-tubulin, a cytoskeleton component critical for centrosome-dependent microtubule nucleation and consequently proper spindle formation and cytokinesis $[28,29]$. A 2 day incubation with 100 nM hsiRNA against B. malayi $\gamma$-tubulin lead to cytokinesis defects during early embryogenesis, as visualized by our optimized immunostaining procedures (Figure 1), and was sufficient to target all zygotic and very early embryonic divisions. Cytokinesis did not occur in zygotes from $\gamma$-tubulin hsiRNA-treated worms resulting in polynucleated 1-cell embryos (Figure 1D, E, F). This suggests that robust phenotypes can be induced by our hsiRNAi procedures at relatively low concentrations of RNA. Similarly, exposure of B. malayi to par-1 hsiRNA $(1 \mu \mathrm{M})$ for 2 days resulted in polarity defective embryos. In C. elegans, the Ser/Thr kinase PAR-1 is crucial for establishing the embryonic anterior-posterior polarity, and PAR-1 removal leads to defects in posterior spindle rotation and loss of division asynchrony at the 2-cell stage [30]. In B. malayi embryos from untreated worms, divisions at the 2-cell stage were asynchronous with the anterior blastomere dividing first and the spindle in the posterior blastomere rotating to align with the anteriorposterior axis (Figure 2A, C) as in C. elegans. However, following exposure to par-1 hsiRNA, divisions at the 2cell stage became synchronous and the spindle in the posterior blastomere failed to rotate (Figure 2B, D), again precisely phenocopying defects assigned to removal of the corresponding protein in C. elegans [30]. In a third example, that of the epithelial cell junction protein AJM-1, we observed a more severe phenotype in B. malayi than in C. elegans. Removal of AJM-1 in C. elegans results in embryonic elongation defects and developmental arrest [31,32]. After targeting the $B$. malayi ajm-1 transcript by RNAi we not only observed robust elongation defects but also severe ventral closure defects and/or epidermal rupture leading to cell protrusions (Figure 3B, C) entirely consistent with disruption of epithelial junction components in C. elegans [32].

The unequivocal phenotypes we report were obtained using hsiRNA concentrations in the range of 0.1 to 1 $\mu \mathrm{M}(\sim 1.3$ to $13 \mu \mathrm{g} / \mathrm{ml})$, whereas other studies addressing RNAi in animal parasitic nematodes have generally used much higher long dsRNA concentrations, frequently in the range 1 to $3.5 \mathrm{mg} / \mathrm{ml}[13,14,20,22,27]$. In the few reports on the use of synthetic short interfering RNA (siRNA) or hsiRNA in animal parasitic nematodes, the concentration has typically ranged from 1 to $5 \mu \mathrm{M}$, with gene silencing observed in some, but not all, cases $[13,22,33]$. It appears that the nematode cuticle may be more permeable to smaller RNA molecules $[22,33]$.

We have confirmed phenotypes for all 7 transcripts targeted by this approach including 6 genes expressed during embryogenesis and 1 in adult tissue, comprising cytoskeleton components, some of them regulators or interactors, as well as genes involved in metabolism. 


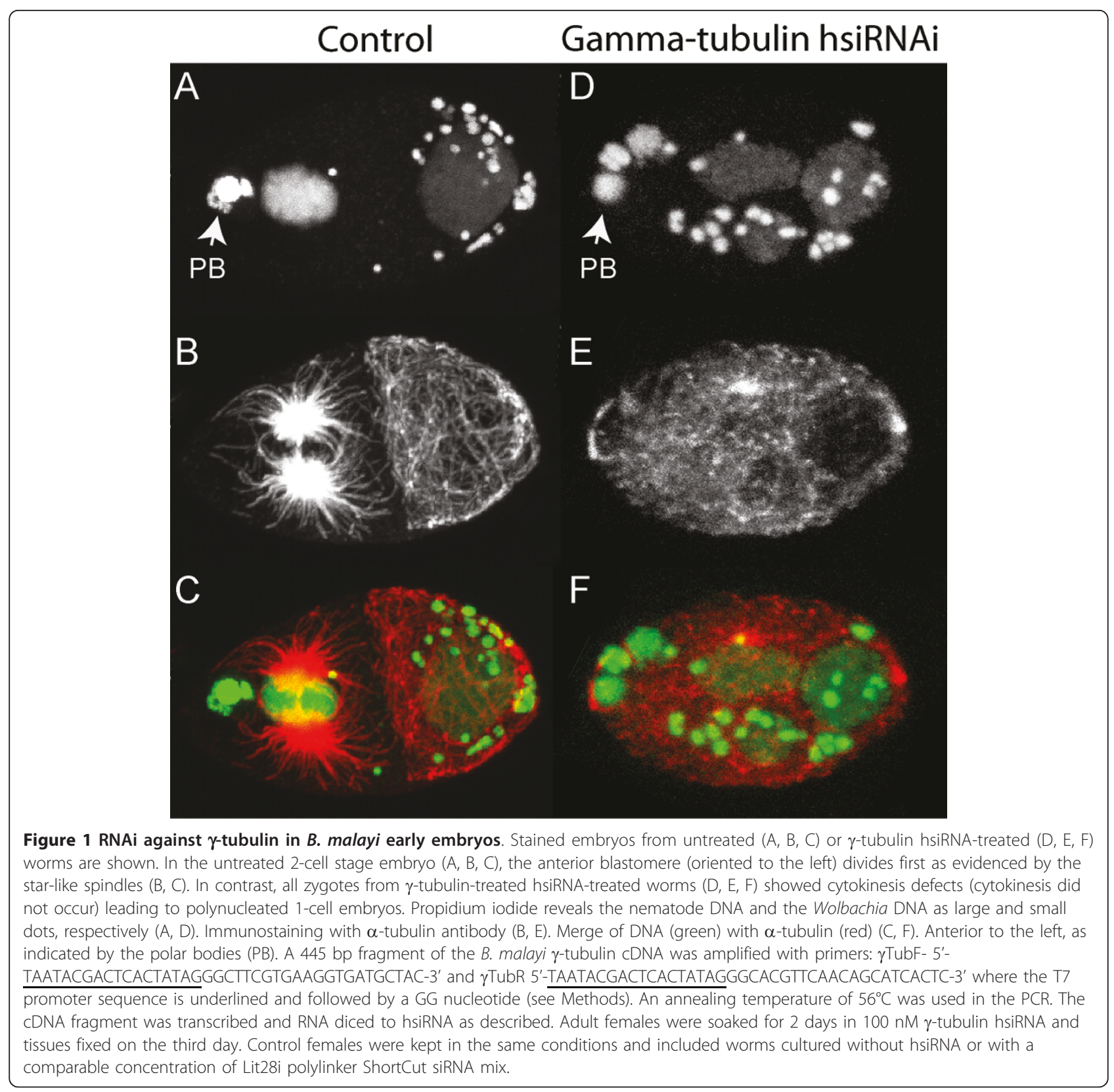

This high rate of effective gene targeting contrasts earlier reports of inefficient RNAi-induced silencing in animal parasitic nematodes [13-18]. Our success may be due in part to targeting genes known to show cellular phenotypes in C. elegans (6 of 7 cases), although in previous studies efficient silencing in $C$. elegans has not translated well into silencing of orthologs in animal parasitic species. For example of 10 genes that show clear larval phenotypes when subjected to RNAi in $C$. elegans, not one resulted in a phenotype when the orthologous genes in Haemonchus contortus orthologs were tested and only two showed any transcript reduction [13]. Our method of RNA delivery may contribute to the effective RNAi results we demonstrate. Most studies have used long dsRNA or one (or a few) synthetic siRNAs that correspond to the gene targeted [14]. Few studies have used hsiRNA mixtures produced enzymatically from longer dsRNA molecules to yield multiple overlapping short RNAs. In one report [22] a variant of the present method was performed using an RNase III digestion protocol which generates predominantly short RNA species ( $13 \mathrm{bp})$ that are not expected to be effective in gene silencing [34]. Unlike other methods employing RNase III, the enzyme conditions used in the present study convert long dsRNA to a mixture of short RNAs ranging from $\sim 18-25 \mathrm{bp}$, predominantly of 


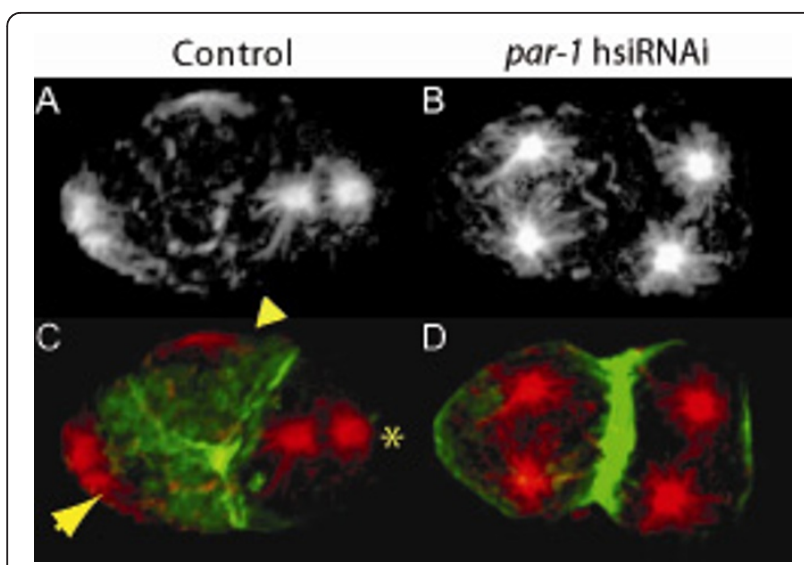

Figure 2 RNAi against par-1 in B. malayi early embryos. Stained embryos from untreated $(A, C)$ or par-1 hsiRNA-treated $(B, D)$ worms are shown. In embryos from untreated control worms $(A, C)$ divisions at the 2-cell stage are asynchronous and the anterior blastomere divides first in a transverse orientation giving rise to anterior blastomere a (ABa; tapered arrowhead) and anterior blastomere $b$ ( $\mathrm{ABb}$; compact arrowhead). The spindle (star-like structure) in the posterior blastomere (P1; asterisk) rotates to align along the anterior-posterior axis. In contrast, in embryos from par- 1 hsiRNA-treated worms (B, D) divisions at the 2-cell stage become synchronous as evidenced by the star-like spindles, and the posterior spindle in $\mathrm{P} 1$ fails to rotate, remaining transverse as in the anterior blastomere. Immunostaining with $\alpha$-tubulin antibody (A, B). Merge of $\alpha$-tubulin (red) with actin (green) as revealed by phalloidin staining $(C, D)$. Anterior to the left. $A \sim 2 \mathrm{~kb}$ fragment of the $B$. malayi par-1 locus (corresponding to $~ 500$ bp cDNA) was amplified with primers: par-1F 5'-TAATACGACTCACTATAGG GGAGAGGA ATCTTGCCAACGG-3' and par-1R 5'-TAATACGAC TCACTATAGG GAACTGCTTGTGCAGATGCGC-3' where the T7 promoter sequence is underlined and followed by a GG nucleotide (see Methods). An annealing temperature of $59^{\circ} \mathrm{C}$ was used in the PCR. The PCR fragment amplified from gDNA was transcribed and RNA diced to hsiRNA as described. Adult females were soaked for 2 days in $1 \mu \mathrm{M}$ par-1 hsiRNA and tissues fixed on the third day. Control females were kept in the same conditions and included worms cultured without hsiRNA or with a comparable concentration of Lit28i polylinker ShortCut siRNA mix.

around the optimal 21 bp size [35]. Furthermore, since all molecules in the mixture are specific for the targeted gene very potent silencing can be achieved. Conversely, off-target effects are minimized since the molar concentration of any molecule in the mixture that matches a non-target transcript will be very low [35].

\section{Conclusions}

By use of optimized immunostaining protocols we have been able to document cellular phenotypes induced by our newly described method for culturing B. malayi in hsiRNA mixtures. Visible cellular defects were observed in all cases tested to date. The entire process can be completed in less than 1 week and is amenable to scaleup, thereby allowing silencing of several genes in parallel. Although RNAi is a proven powerful tool for

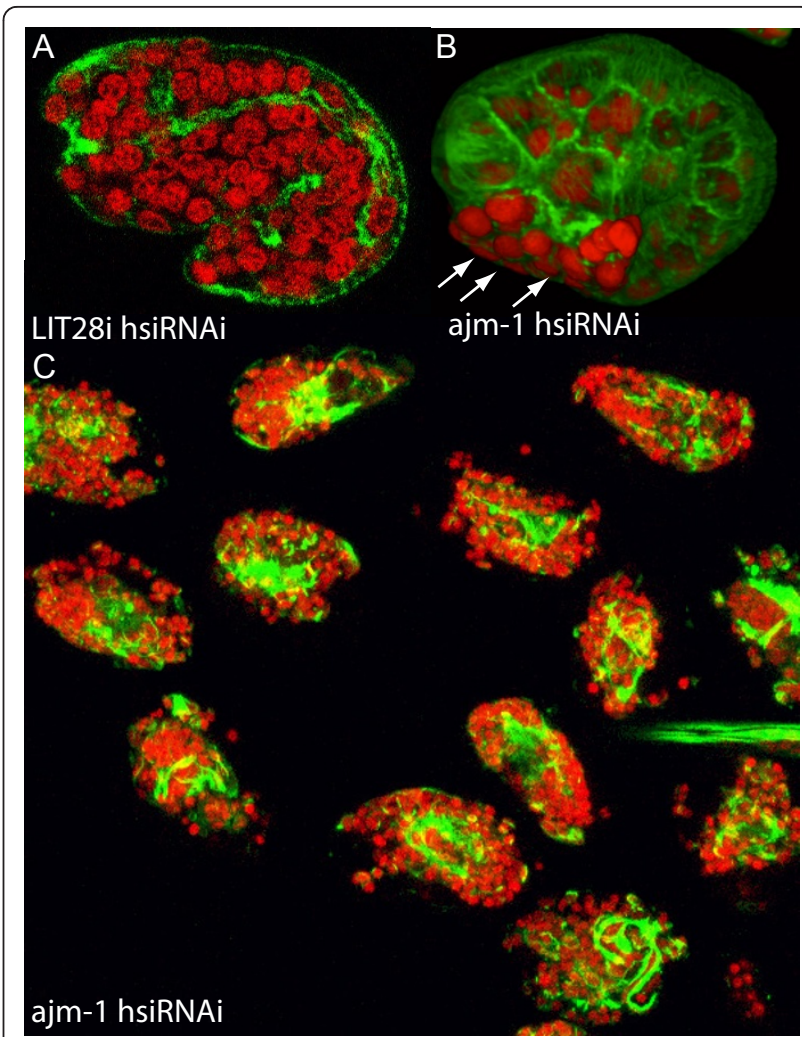

Figure 3 RNAi against ajm-1 in B. malayi early embryos. Stained embryos from control Lit28i hsiRNA-treated (A) or ajm-1 hsiRNAtreated $(B, C)$ worms are shown. RNAi control 1.5 fold embryo (A). For comparison, a 1.5 fold embryo displaying morphogenesis defects as a result of loss of AJM-1 (B). The extruded cells (arrows) indicate a compromised junctional integrity of the epidermis. A cluster of late-stage embryos extracted from a uterine fragment, showing robust and consistent phenotypes of cell extrusion and elongation defects (C). Staining with propidium iodide (red) and phalloidin (green) indicate DNA and actin, respectively. A 442 bp fragment of the $B$. malayi ajm-1 CDNA was amplified with primers: ajmF- 5'-TAATACGACTCACTATAGGGGAACGACTATATGTACGGTG -3' and ajmR 5'-TAATACGACTCACTA TAGGGGATCATATTGACG ACACAGAG $-3^{\prime}$ where the T7 promoter sequence is underlined and followed by a GG nucleotide (see Methods). An annealing temperature of $60^{\circ} \mathrm{C}$ was used in the PCR. The CDNA fragment was transcribed and RNA diced to hsiRNA as described. Adult females were soaked for 2 days in $1 \mu \mathrm{M}$ ajm-1 hsiRNA and tissues fixed on the third day. Control females were kept in the same conditions and included worms cultured without hsiRNA or with a comparable concentration of Lit28i polylinker ShortCut siRNA mix.

investigating gene function in many diverse organisms [36], including the model nematode C. elegans where it has been used for high-throughput functional genomics $[12,37]$, the technology had previously met with limited success when applied to animal parasitic nematodes [13-18].

The combination of RNAi and immunostaining we report provide critical tools for characterizing 
developmental processes and unraveling the complex association and transmission pattern of Wolbachia endosymbionts in filarial nematodes. More generally, our RNAi methods can be used to investigate components of metabolic pathways in B. malayi as well as the function of genes that are either absent or very divergent in the human genome to validate candidate drug targets for subsequent development [38]. Besides our immunofluorescence-based phenotype analysis, RNAi-mediated transcript knockdown can also be confirmed by RT-PCR or monitoring of readily scored phenotypes such as microfilarial output or molting efficiency [21,22]. The general conservation of structure and basic biology of all nematodes raises the exciting prospect that our approach can be deployed with little or no modification to any species amenable to brief in vitro maintenance and facilitate a similarly robust reverse genetic approach towards characterization of gene function in some of the worlds most devastating pathogens.

\section{Additional material}

Additional file 1: Detailed RNAi and immunostaining protocol. An enumerated step-by-step protocol with helpful tips.

Additional file 2: Handling B. malayi worms and set-up of the culture plate. Worms are handled under a laminar flow hood using a curved pick and placed in $1 \mathrm{ml}$ WCM (A, B). For multiple hsiRNAi experiments, the 2 worms in each well corresponding to 1 experimental condition can be moved to another plate, in the same corresponding well (C). For a single hsiRNAi experiment over 3 days or less, see Additional file 3 .

Additional file 3: Use of a 12-well plate for one hsiRNAi experiment with a matched non-treated control. At day 1, the plate is divided in two, with the left part used for hsiRNAi, and the right part used for the control worms. The hsiRNA (i.e. $1 \mu \mathrm{M}$ or $\sim 13.5 \mu \mathrm{g}$ ) is added to the first well. Both treated and control wells receive $1 \mathrm{ml}$ of WCM, then 2 worms as described above. Purple arrows show how the worms are moved every 12 hours to different wells with fresh medium, supplemented with hsiRNA in the case of the treated worms. For larger experiments with several samples, worms are transferred to corresponding wells of new culture plates every $12 \mathrm{hr}$.

Additional file 4: Preparing worm samples for fixation. Worms are placed on a glass slide using a curved pick. A razor blade is used to cut the adult worms into smaller fragments (A). This can be monitored under a dissecting microscope. Embryos and adult tissues are transferred by directing the flow of buffer towards a corner of the glass slide and into a microcentrifuge tube (B).

\section{Acknowledgements}

We thank Nonglak Supakorndej of TRS labs for skillfully harvesting and sexing B. malayi worms, and George Tzertzinis for critical reading of the manuscript. This work was supported by a grant awarded to The Liverpool School of Tropical Medicine by the Bill and Melinda Gates Foundation (AWOL consortium) and by New England Biolabs.

\section{Author details}

'Department of Molecular, Cell and Developmental Biology, University of California Santa Cruz, 1156 High Street, Santa Cruz, CA 95604, USA. ${ }^{2}$ Division of Molecular Parasitology, New England Biolabs, Inc., 240 County Road, Ipswich, MA 01938, USA.

\section{Authors' contributions}

FL participated in study conception and design, generated RNA, performed the RNAi and immunostaining, and helped draft and edit the manuscript. JF participated in study conception and design, generated RNA, CDNA, and drafted the manuscript. BS participated in study design and helped draft and edit the manuscript. WS participated in study conception and design, and helped draft and edit the manuscript. All authors read and approved the final manuscript.

\section{Competing interests}

The authors declare that they have no competing interests.

Received: 14 October 2011 Accepted: 13 January 2012

Published: 13 January 2012

\section{References}

1. Bourguinat C, Pion SD, Kamgno J, Gardon J, Duke BO, Boussinesq M, Prichard RK: Genetic selection of low fertile Onchocerca volvulus by ivermectin treatment. PLoS Negl Trop Dis 2007, 1:e72.

2. Osei-Atweneboana MY, Awadzi K, Attah SK, Boakye DA, Gyapong JO, Prichard RK: Phenotypic Evidence of Emerging Ivermectin Resistance in Onchocerca volvulus. PLoS Negl Trop Dis 2011, 5:e998.

3. Osei-Atweneboana MY, Eng JK, Boakye DA, Gyapong JO, Prichard RK: Prevalence and intensity of Onchocerca volvulus infection and efficacy of ivermectin in endemic communities in Ghana: a two-phase epidemiological study. Lancet 2007, 369:2021-2029.

4. Taylor MJ, Bandi C, Hoerauf A: Wolbachia bacterial endosymbionts of filarial nematodes. Adv Parasitol 2005, 60:245-284.

5. Taylor MJ, Hoerauf A, Bockarie M: Lymphatic filariasis and onchocerciasis. Lancet 2010, 376:1175-85.

6. Landmann F, Foster JM, Slatko B, Sullivan W: Asymmetric Wolbachia segregation during early Brugia malayi embryogenesis determines its distribution in adult host tissues. PLoS Negl Trop Dis 2010, 4:e758.

7. Ghedin E, Wang S, Spiro D, Caler E, Zhao Q, Crabtree J, Allen JE, Delcher AL, Guiliano DB, Miranda-Saavedra D, et al: Draft genome of the filarial nematode parasite Brugia malayi. Science 2007, 317:1756-1760

8. Foster J, Ganatra M, Kamal I, Ware J, Makarova K, Ivanova N, Bhattacharyya A, Kapatral V, Kumar S, Posfai J, et al: The Wolbachia genome of Brugia malayi: endosymbiont evolution within a human pathogenic nematode. PLoS Biol 2005, 3:e121

9. Burglin TR, Lobos E, Blaxter ML: Caenorhabditis elegans as a model for parasitic nematodes. Int J Parasitol 1998, 28:395-411.

10. Vanfleteren JR, Van de Peer Y, Blaxter ML, Tweedie SA, Trotman C, Lu L, Van Hauwaert ML, Moens L: Molecular genealogy of some nematode taxa as based on cytochrome $\mathrm{c}$ and globin amino acid sequences. Mol Phylogenet Evol 1994, 3:92-101.

11. Geary TG, Thompson DP: Caenorhabditis elegans: how good a model for veterinary parasites? Vet Parasitol 2001, 101:371-386

12. Kamath RS, Fraser AG, Dong Y, Poulin G, Durbin R, Gotta M, Kanapin A, Le Bot N, Moreno S, Sohrmann M, et al: Systematic functional analysis of the Caenorhabditis elegans genome using RNAi. Nature 2003, 421:231-237.

13. Geldhof P, Murray L, Couthier A, Gilleard JS, McLauchlan G, Knox DP, Britton C: Testing the efficacy of RNA interference in Haemonchus contortus. Int J Parasitol 2006, 36:801-810.

14. Geldhof P, Visser A, Clark D, Saunders G, Britton C, Gilleard J, Berriman M, Knox D: RNA interference in parasitic helminths: current situation, potential pitfalls and future prospects. Parasitology 2007, 134:609-619.

15. Knox DP, Geldhof $P$, Visser $A$, Britton C: RNA interference in parasitic nematodes of animals: a reality check? Trends Parasitol 2007, 23:105-107.

16. Lendner M, Doligalska M, Lucius R, Hartmann S: Attempts to establish RNA interference in the parasitic nematode Heligmosomoides polygyrus. Mol Biochem Parasitol 2008, 161:21-31.

17. Viney ME, Thompson FJ: Two hypotheses to explain why RNA interference does not work in animal parasitic nematodes. Int J Parasitol 2008, 38:43-47.

18. Visser A, Geldhof P, de Maere V, Knox DP, Vercruysse J, Claerebout E: Efficacy and specificity of RNA interference in larval life-stages of Ostertagia ostertagi. Parasitology 2006, 133:777-783.

19. Dalzell JJ, McVeigh P, Warnock ND, Mitreva M, Bird DM, Abad P, Fleming CC, Day TA, Mousley A, Marks NJ, et al: RNAi Effector Diversity in Nematodes. PLoS Negl Trop Dis 2011, 5:e1176. 
20. Aboobaker AA, Blaxter ML: Use of RNA interference to investigate gene function in the human filarial nematode parasite Brugia malayi. Mol Biochem Parasitol 2003, 129:41-51.

21. Ford L, Guiliano DB, Oksov Y, Debnath AK, Liu J, Williams SA, Blaxter ML, Lustigman S: Characterization of a novel filarial serine protease inhibitor, Ov-SPI-1, from Onchocerca volvulus, with potential multifunctional roles during development of the parasite. J Biol Chem 2005, 280:40845-40856.

22. Ford L, Zhang J, Liu J, Hashmi S, Fuhrman JA, Oksov Y, Lustigman S: Functional analysis of the cathepsin-like cysteine protease genes in adult Brugia malayi using RNA interference. PLoS Negl Trop Dis 2009, 3: e377.

23. Lustigman S, Zhang J, Liu J, Oksov Y, Hashmi S: RNA interference targeting cathepsin $L$ and $Z$-like cysteine proteases of Onchocerca volvulus confirmed their essential function during L3 molting. Mol Biochem Parasitol 2004, 138:165-170.

24. Pfarr K, Heider U, Hoerauf A: RNAi mediated silencing of actin expression in adult Litomosoides sigmodontis is specific, persistent and results in a phenotype. Int I Parasitol 2006, 36:661-669.

25. Song C, Gallup JM, Day TA, Bartholomay LC, Kimber MJ: Development of an in vivo RNAi protocol to investigate gene function in the filarial nematode, Brugia malayi. PLoS Pathog 2010, 6:e1001239.

26. Tachu B, Pillai S, Lucius R, Pogonka T: Essential role of chitinase in the development of the filarial nematode Acanthocheilonema viteae. Infect Immun 2008, 76:221-228.

27. Samarasinghe $B, K$ nox DP, Britton C: Factors affecting susceptibility to RNA interference in Haemonchus contortus and in vivo silencing of an H11 aminopeptidase gene. Int J Parasitol 2011, 41:51-59.

28. Hannak E, Oegema K, Kirkham M, Gonczy P, Habermann B, Hyman AA: The kinetically dominant assembly pathway for centrosomal asters in Caenorhabditis elegans is gamma-tubulin dependent. J Cell Biol 2002, 157:591-602

29. Joshi HC, Palacios MJ, MCNamara L, Cleveland DW: Gamma-tubulin is a centrosomal protein required for cell cycle-dependent microtubule nucleation. Nature 1992, 356:80-83.

30. Kemphues KJ, Priess JR, Morton DG, Cheng NS: Identification of genes required for cytoplasmic localization in early C. elegans embryos. Cell 1988, 52:311-320.

31. Koppen M, Simske JS, Sims PA, Firestein BL, Hall DH, Radice AD, Rongo C Hardin JD: Cooperative regulation of AJM-1 controls junctional integrity in Caenorhabditis elegans epithelia. Nat Cell Biol 2001, 3:983-991.

32. Lynch AM, Hardin J: The assembly and maintenance of epithelial junctions in C. elegans. Front Biosci 2009, 14:1414-1432.

33. Issa Z, Grant WN, Stasiuk S, Shoemaker CB: Development of methods for RNA interference in the sheep gastrointestinal parasite, Trichostrongylus colubriformis. Int J Parasitol 2005, 35:935-940.

34. Elbashir SM, Martinez J, Patkaniowska A, Lendeckel W, Tuschl T: Functional anatomy of siRNAs for mediating efficient RNAi in Drosophila melanogaster embryo lysate. EMBO J 2001, 20:6877-6888.

35. Morlighem JE, Petit C, Tzertzinis G: Determination of silencing potency of synthetic and RNase III-generated siRNA using a secreted luciferase assay. Biotechniques 2007, 42:599-606.

36. Liu Q, Paroo Z: Biochemical principles of small RNA pathways. Annu Rev Biochem 2010, 79:295-319.

37. Simmer F, Moorman C, van der Linden AM, Kuijk E, van den Berghe PV, Kamath RS, Fraser AG, Ahringer J, Plasterk RH: Genome-wide RNAi of $C$. elegans using the hypersensitive rrf-3 strain reveals novel gene functions. PLOS Biol 2003, 1:e12.

38. Behm CA, Bendig MM, McCarter JP, Sluder AE: RNAi-based discovery and validation of new drug targets in filarial nematodes. Trends Parasitol 2005, 21:97-100.

doi:10.1186/1756-3305-5-16

Cite this article as: Landmann et al: Efficient in vitro RNA interference and immunofluorescence-based phenotype analysis in a human parasitic nematode, Brugia malayi. Parasites \& Vectors 2012 5:16.

\section{Submit your next manuscript to BioMed Central and take full advantage of:}

- Convenient online submission

- Thorough peer review

- No space constraints or color figure charges

- Immediate publication on acceptance

- Inclusion in PubMed, CAS, Scopus and Google Scholar

- Research which is freely available for redistribution 